\title{
Anforderungsmanagement für die modellbasierte Entwicklung mechatronischer Systeme im digitali- sierten und vernetzten Umfeld
}

\author{
Or Aviv Yarom ${ }^{1 *}$, Jie Zhang ${ }^{1}$, Christian Raulf ${ }^{2}$, Xiaobo Liu-Henke ${ }^{1}$, Thomas Vietor ${ }^{2}$ \\ ${ }^{1}$ Institut für Mechatronik, Ostfalia Hochschule für angewandte Wissenschaften, Salzdahlumer Str. 46/48, \\ 38302 Wolfenbüttel, Deutschland; *o.yarom@ostfalia.de \\ IInstitut für Konstruktionstechnik, Technische Universität Braunschweig, Hermann-Blenk-Str. 42 \\ 38108 Braunschweig, Deutschland
}

\begin{abstract}
Der folgende Beitrag beschreibt eine Entwurfsmethodik für die modellbasierte Entwicklung mechatronischer Systeme im digitalisierten und vernetzten Umfeld, unter Anwendung eines modellbasierten Anforderungsmanagements. Das Ziel ist eine ganzheitliche Betrachtung komplexer Systeme und derer Schnittstellen zwischen dynamischen Anforderungen und Systemstruktur. Im weiteren Verlauf wird das Vorgehen anhand eines Beispiels aus der Fertigungstechnik aufgezeigt und validiert.
\end{abstract}

\section{Einleitung}

Gesellschaft und Wirtschaft befinden sich aufgrund der zunehmenden Digitalisierung und Vernetzung in einem tiefgreifenden Wandel. Ein Beispiel hierfür ist die Automobilindustrie, die unter Einsatz des autonomen Fahrens und der Herausforderung eines flexiblen, anwendungsspezifischen Fahrzeugeinsatzes mit rasant steigenden Anforderungen an Fahrzeugentwicklung und Software zu kämpfen hat. Ein weiteres Beispiel ist die Fertigungsindustrie, die zur Erhaltung ihrer Wettbewerbsfähigkeit bei steigender Anzahl an Produktvariationen auf Industrie 4.0 (I4.0)-Lösungen zur Produktivitäts- und Flexibilitätssteigerung angewiesen ist. Dies sind nur zwei ausgewählte Domänen in denen sich der Bedarf an leistungsfähigen Komponenten aus den Disziplinen Mechanik, Elektrik/Elektronik und Informationstechnik bei der Entwicklung innovativer Produkte bzw. Systeme kontinuierlich erhöht. Das Einbringen moderner Kommunikationstechnologien ermöglicht die Vernetzung von Systemen untereinander, mit deren (System-)Umgebung oder auch mit dem Internet of Things (IoT) und schafft zusätzliche Mehrwerte für deren Nutzung und Anwendung. Darüber hinaus erhöhen komplexe Algorithmen, z.B. aus dem Gebiet der künstlichen Intelligenz (KI), den notwendigen Aufwand für den Entwurf und die Absicherung der resultierenden cyberphysischen Systeme (CPS) massiv. Daher erfordert die Entwicklung und Validierung komplexer und intelligenter Systeme in einer zunehmend schnelllebigen, digitalisierten und vernetzten Umgebung die Umgestaltung bewährter Methoden und Prozesse.

Die vom Europäischen Fonds für regionale Entwicklung (EFRE) geförderten Innovationsverbunde autoMoVe (Dynamisch konfigurierbare Fahrzeugkonzepte für den nutzungsspezifischen autonomen Fahrbetrieb) und Synus (Methoden und Werkzeuge für die synergetische Konzipierung und Bewertung von Industrie 4.0-Lösungen) befassen sich intensiv mit etwaigen Forschungsfragen in den Domänen automatisiertes Fahren und I4.0.

Dieser Beitrag ensteht im Rahmen dieser beiden Forschungsprojekte und befasst sich mit einer ganzheitlichen Methodik für den Entwurf intelligenter mechatronischer Systeme, vom frühen Stadium der Anforderungserhebung, bis hin zur Systemabsicherung. Die Methodik wird an einem Anwendungsbeispiel zur konfliktfreien Trajektorienplanung demonstriert und validiert.

\section{Stand des Wissens}

In diesem Kapitel werden zunächst bewährte sowie moderne Entwurfsmethoden aus der Konstruktionssystematik und Mechatronikforschung vorgestellt.

\subsection{Modellbasiertes \\ Anforderungsmanagement}

Digitale, vernetzte mechatronische Produkte zeichnen sich dadurch aus, dass die gewünschten Fähigkeiten oder 
Features nicht einem einzelnen Bauteil oder einer Baugruppe zugeordnet werden können, sondern ein komplexes Zusammenspiel vieler Komponenten aus verschiedenen Domänen erfordern. Dabei führt der Einsatz von immer mehr Softwarekomponenten zu einem Anstieg der Komplexität der technischen Systeme. Ein mechatronisches System beinhaltet dabei Elemente aus den Bereichen Mechanik, Elektronik und Informatik, beschreibt deren Zusammenwirken und grenzt sie gegenüber ihrer Umgebung ab. [1] Das zu entwickelnde System, das sogenannte Sytem of Interest (SOI) lässt sich, in Teil- oder Subsysteme untergliedern und kann wiederum selbst in ein übergeordnetes System (komplexe Systemumgebung) eingebettet werden. [2]

Das des Systems Engineering beschäftigt sich mit der interdisziplinären Entwicklung und Umsetzung technischer Systeme. Hierbei gilt die Annahme, dass das System nicht bloß die Summe seiner Elemente ist, sondern sich über deren Zusammenhänge definiert. [3] Existiert nun ein globales abstraktes Metamodell, welches die $\mathrm{Zu}-$ sammenhänge und Wechselwirkungen der einzelnen Elemente beschreibt, spricht man vom Model Based Systems Engineering (MBSE). Dieses Metamodell soll im Folgenden als Systemmodell bezeichnet werden.

In der Konstruktionstechnik dient das Model-based Systems Engineering unter anderem dazu, verschiedene Entwicklungsaktivitäten in einem zentralen interdisziplinären Systemmodell zu dokumentieren und zu verwalten. Dieses kann z.B. genutzt werden, um kontextspezifische Sichten für die verschiedenen Entwicklungsdomänen zu generieren oder Systemabhängigkeiten und -zusammenhänge zu erkennen und zu visualisieren [2]. Um die komplexen Systemzusammenhänge zu modellieren, bedient man sich der grafischen Modellierungssprache SysML. Die wesentlichen Säulen der Systemmodellierung mit SysML werden durch die Darstellung in den drei Bereichen Anforderungen, Systemverhalten und der Systemstruktur gebildet. [4]

$\mathrm{Zu}$ Beginn eines jeden Entwicklungsprozesses gilt es die Anforderungen an das zu entwickelnde System zu erheben und zu dokumentieren. Die Anforderungen selbst stammen in erster Linie aus den Bedürfnissen der Stakeholder. Ergänzend fließen geltende Normen, Richtlinien und gesetzliche Rahmenbedingungen ein sowie Erfahrungswerte aus bereits vorhanden Systemen. [5] Um die Anforderungen auch in komplexen Systemen umfassend zu erfassen und zu verwalten, wurde bisher eine Gliederung in die Betrachtungsebenen "Geschäftsebene”, "Systemebene” und “Komponentenebene” vorgenommen.
Zwischen den Anforderungen und der Systemarchitektur bestehen Wechselwirkungen: Die Architektur gründet sich auf den entsprechenden Anforderungen, gleichzeitig wirken sich Entwurfsentscheidungen auf die Anforderungen aus. [6] Anforderungen sind somit nicht bloß statisch, zu Beginn der Entwicklung definiert, sondern können dynamisch verändert und angepasst werden. [5] Insbesondere vor dem Gesichtspunkt interdisziplinärer Zusammenarbeit erschwert dieser Umstand den Entwicklungsprozess. Das Anforderungs- oder RequirementsManagement mittels SysML wird als modellbasiertes Anforderungsmanagement (MBRE) bezeichnet. MBRE bietet die Möglichkeit, dynamische Anforderungen über den gesamten Produktentehungsprozess bzw. -lebenszyklus im Systemmodell zu verwalten und Änderungen eindeutig zu kommunizieren und nachzuverfolgen.

\subsection{Mechatronische Entwurfsmethodik}

Die in der Mechatronikforschung bewährte Entwurfsmethodik basiert auf einer Modularisierung und Hierarchisierung des komplexen Gesamtsystems. Dabei wird das SOI in einem Top-Down Verfahren zunächst in intelligente, gekapselte Module aus mechatronischen Teilsystemen mit definierten Schnittstellen zur Kommunikation mit ihrer Umgebung zerlegt. Anschließend werden die Module in einer hierarchischen Struktur in Beziehung zueinander gesetzt. [7] Abbildung 1 zeigt exemplarisch die hierarchische Struktur des Forschungsfahrzeugs FREDY (Funktionsträger für regenerative Elektromobilität und Fahrdynamik) auf sechs Hierarchieebenen.

Die mechatronischen Funktionsmodule (MFM) bilden die unterste und zugleich vitalste Hierarchieebene. Sie beinhalten nicht weiter teilbare Module, bestehend aus Grundaufbau, Sensorik, Aktorik und einer grundlegenden Informationsverarbeitung. Sie besitzen physikalische und informatorische Schnittstellen zu den überlagerten mechatronischen Funktionsgruppen (MFG). Diese besitzen keine eigene Aktorik, sondern greifen auf die MFM zu und realisieren in Kombination mit einer eigenen Informationsverarbeitung höherwertige Funktionen. Mehrere MFM und MFG, bilden in ihrer Gesamtheit ein autonomes mechatronisches System (AMS). Ein AMS ist unabhängig von seiner Umgebung und verfügt über eine eigene Informationsverarbeitung und Informationsschnittstellen zu anderen Systemen. Wenn mehrere AMS Informationen durch digitale Vernetzung austauschen und so bspw. kooperativ operieren spricht man von auto- 

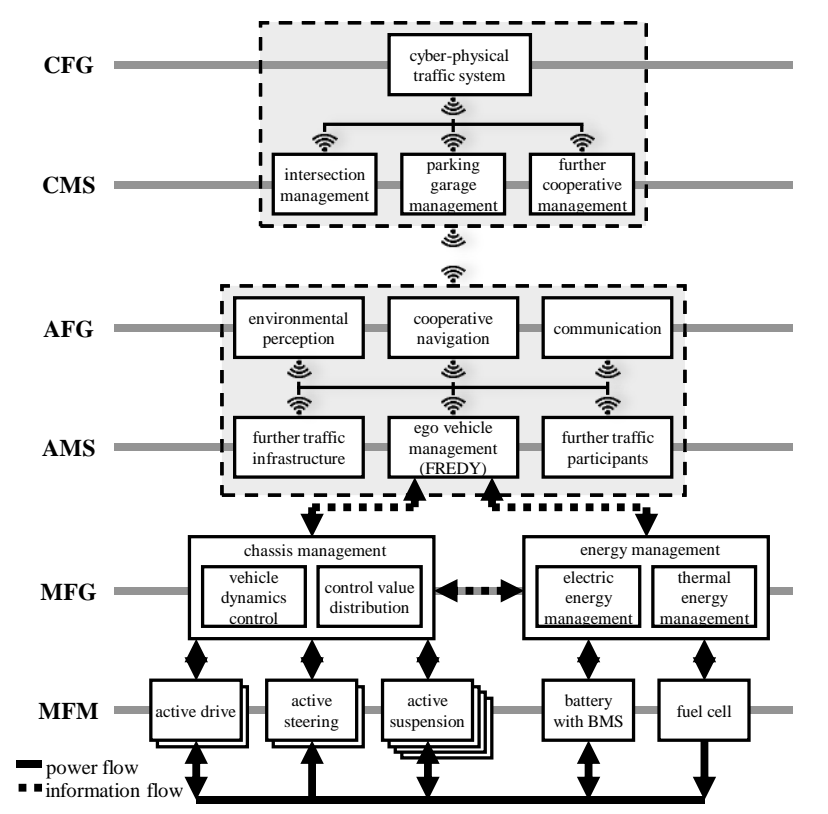

Abbildung 1: Mechatronische Strukturierung des FREDY

nome Funktionsgruppen (AFG). Agieren die AFG in einem speziellen Bereich, wie einem Kreuzungsmanagement, werden Entscheidungen in einem vernetzten mechatronischen System (CMS) getroffen und koordiniert. Das CMS reguliert den Informationsfluss und leitet entsprechende Befehle an unterlagerte AFG und AMS weiter. Mehre CMS lassen sich zu vernetzen Funktionsgruppen (CFG) zusammenfassen, sodass Daten in strukturierten Clustern ausgetauscht werden können.

Die anschließende Auslegung jedes Funktionsmoduls mit der zugehörigen Informationsverarbeitung erfolgt Bottom-Up unter Anwendung des mechatronischen Entwicklungskreises [8]. In Kombination mit der anschließenden Absicherung und Integration wird dieses Vorgehen als mechatronische Komposition bezeichnet. Basierend auf den Anforderungen und der Spezifikation werden die physikalische und mathematische Modellierung sowie die Analyse und Parameteridentifikation durchgeführt, so dass ein validiertes Modell des Systemverhaltens die Grundlage für die modellbasierte Entwicklung von intelligenten Informationsverarbeitungen bildet. Diese Methodik zeichnet sich durch eine frühzeitige Validierung und Verifikation aus, so dass Entwicklungszeit und -kosten reduziert werden können [8]. Die Reglerentwicklung erfolgt nach dem Rapid Control Prototyping (RCP) in einem vollständig verifikationsorientierten Prozess mit MiL-, SiL- und HiL-Simulationen.

\section{Kopplung von Anforderungs- management und mechatroni- scher Entwurfsmethodik}

\subsection{Problemstellung und Motivation}

Die in Kapitel 1 vorgestellten Entwurfsmethoden befassen sich mit der strukturierten Entwicklung intelligenter mechatronischer Systeme, die wegen ihrer zahlreichen miteinander agierenden Komponenten und der digitalen Vernetzung mit ihrer Umwelt hochkomplex sind. Aufgrund ihres Ursprungs aus den Domänen der Konstruktionstechnik und der Mechatronikforschung bedienen die Entwurfsmethoden jeweils unterschiedliche Sichtweisen und Schwerpunkte. Das MBRE beschreibt Systemzusammenhänge und Anforderungen auf einer übergeordneten Metaebene als essentielles Werkzeug zur Ermittlung, Dokumentation und Verwaltung von Anforderungen. Es eignet sich sehr gut, um sich ändernde Anforderungen und deren Auswirkungen im komplexen Gesamtsystem zu überblicken und zu verfolgen. Der dynamische Charakter und damit der entscheidende Vorteil des MBRE geht allerdings verloren, wenn das Anforderungsmanagement von der konkreten Entwicklungstätigkeit entkoppelt wird. In diesem Fall müssen die Anforderungen in natürlicher Sprache, klassischerweise in Form von Lasten- oder Pflichtenheften, an beteiligte Entwickler weitergeben werden. Der mechatronische Entwurf hingegen fokussiert eher die funktionalen und strukturellen Wechselwirkungen des Systems, die für den anschließenden konkreten Entwurfs- und Absicherungsprozess elementar sind. Man ist sowohl bei der Modularisierung und Hierarchisierung als auch bei der Auslegung jedes Funktionsmoduls auf Anforderungen angewiesen. Zum einen begründet sich die mechatronische Strukturierung auf den Anforderungen. Zum anderen beeinflussen die konzeptionellen Entscheidungen zur Strukturierung aber auch die Anforderungen durch Einschränkungen oder neue Ausprägungen. Gleichzeitig gilt, dass die Komplexität der Anforderungen mit der Komplexität des Systems steigt. Dadurch entstehen Zusammenhänge und Abhängigkeiten, die insbesondere bei interdisziplinären Entwicklungstätigkeiten kaum überschaubar sind. Treten zusätzlich dynamische Anforderungen auf, ergibt sich bei klassischem Lastenheft-basierten Anforderungsmanagement eine Quelle für schwerwiegende Fehler.

Obwohl beide Entwurfsmethoden in ihrer jeweiligen Domäne etabliert sind, besitzen sie Schwächen in der 
Schnittstelle zwischen dynamischen Anforderungen und Systemstruktur. Da beide Methoden diese Schnittstelle bedienen, ist es zielführend sie miteinander zu verkoppeln. Dadurch entsteht eine ganzheitliche und durchgängige Entwurf Methodik für komplexe mechatronische Systeme im digitalisierten und vernetzten Umfeld.

\subsection{Konzept und Anforderungen}

Das MBRE eines Systems lässt sich aus zwei Perspektiven umsetzen: Struktur- und Funktionsperspektive. In der Strukturperspektive werden strukturelle Zusammenhänge und Abhängigkeiten des Systems bzw. dessen Anforderungen aufgeführt. Somit bildet die Strukturperspektive die erste Schnittstelle zur mechatronischen Strukturierung im mechatronischen Entwurf. Um die dynamischen Anforderungen und ihre Auswirkungen auf die mechatronische Strukturierung zu verknüpfen, bedarf es einer gemeinsamen Darstellungs- / Betrachtungsform.

In den fortgeschrittenen Phasen des mechatronischen Entwurfs werden die funktionalen Schnittstellen und $\mathrm{Zu}$ sammenhänge einzelner Funktionsmodule festgelegt. Die sogenannte Funktionsstruktur beschreibt dabei, welche Ein- und Ausgangsgrößen das zu entwickelnde Modul besitzt und wie es mit anderen Modulen wechselwirkt. Aus der Funktionsperspektive des MBRE adressieren die Funktionsmodule auf höherer Ebene das gewünschte Verhalten des SOI. Gleichzeitig werden auch Anforderungen an die Funktionsmodule und deren Schnittstellen abgebildet. Zum einen bilden diese Anforderungen im Rahmen der mechatronischen Komposition die Grundlage für den Detailentwurf. Zum anderen dienen sie als Vergleichsmaß bei der Absicherung.

Die Kopplung des MBRE mit der mechatronischen Entwurfsmethodik soll Durchgängigkeit infolge einer ganzheitlichen Betrachtung von Anforderungen, Struktur, Funktionen und Absicherung des SOI schaffen. Um die Durchgängigkeit zu gewährleisten, ist es erforderlich diese Zusammenhänge in einem Systemmodell abzubilden und zu verknüpfen. Die Implementierung muss dabei so erfolgen, dass Änderungen von Anforderungen und Struktur möglich sind. Die Auswirkungen, der dynamischen Anforderungen des Gesamtsystems oder einzelner Systembestandteile auf ihre jeweilige Umgebung, müssen ebenfalls abgebildet werden. Das bedeutet, dass Änderungen bzw. Änderungsketten mithilfe des Systemmodells über die gesamte System- und Funktionsstruktur mindestens nachvollziehbar sein müssen. So können Ent- wicklungspartner, die an demselben oder einem angrenzenden Systembestandteil arbeiten zur Fehlervermeidung informiert werden. Idealerweise lassen sich die Änderungen nicht nur nachverfolgen, sondern durch eine parametrische Implementierung der Anforderungen automatisch an die angrenzenden, abhängigen oder betroffen Systembestandteile weitergeben. Somit werden Entwicklungspartner nicht nur über Änderungen informiert, sondern es erfolgt eine automatische Aktualisierung der eigenen Anforderungen. Dies erfordert allerdings die Implementierung einer entsprechenden Reglementierung für die Weitergabe im Systemmodell. Im Rahmen der Reglementierung ist auch eine Konsistenzprüfung und Priorisierung der Anforderungen sinnvoll.

\subsection{MBRE-basierte mechatronische Entwurfs- methodik}

Ausgehend vom Stand des Wissens (Kapitel 1) sowie dem Konzept und den Anforderungen (Abschnitt 2.2), wird in diesem Abschnitt die MBRE-basierte mechatronische Entwurfsmethodik (Abbildung 2) für die modellbasierte Entwicklung mechatronischer Systeme im digitalisierten und vernetzten Umfeld vorgestellt.

Für Anforderungen und Struktur gilt allgemein, dass sich die im Laufe der Entwurfsphase zunächst vom Abstrakten zum Konkreten entwickeln. Während des Detailentwurfs und der anschließenden Absicherung im Rahmen der mechatronischen Komposition werden einzelne Systembestandteile sukzessive zum Gesamtsystem integriert, sodass der Detaillierungsgrad wieder sinkt. Ausgangspunkt sind in jedem Entwicklungsprozess die Kundenbedürfnisse auf der obersten Abstraktionsebene.

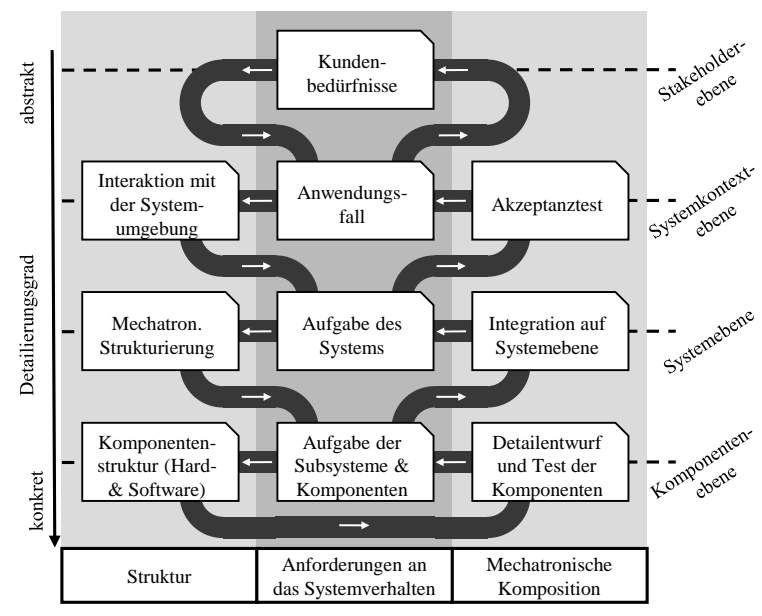

Abbildung 2: Schematische Darstellung der MBRE-basierte mechatronische Entwurfs-methodik 
Diese stehen hier stellvertretend für sämtliche Stakeholder-Anforderungen. Die Stakeholderebene beschreibt folglich nicht nur Nutzerbedürfnisse, sondern auch übergeordnete Anforderungen, wie Kosten, Qualität, Nachhaltigkeit, usw. Unter weiterer Betrachtung von Normen, gesetzlichen Richtlinien oder auch Erfahrungswerten aus bestehenden Systemen, lassen sich Anwendungsfälle definieren, die auf das gewünschte Verhalten des SOI auf der Systemkontextebene abzielen. Daraus lassen sich Art, Gegenstand und Wirkung der Interaktion des SOI mit seiner Umgebung ableiten. Auf Grundlage dessen bestimmt sich die konkrete Aufgabe des SOI, die in Form von Anforderungen auf der Systemebene beschrieben wird. Hieraus kann nun wiederum eine hierarchische mechatronische Strukturierung (Abbildung 1) für das SOI abgeleitet werden. Anschließend müssen auf der Komponentenebene Anforderungen für jedes gekapselte Funktionsmodul definiert werden. Daraus entsteht eine Funktionsstruktur, die beschreibt welche Schnittstellen das zu entwickelnde Modul besitzt und wie es mit anderen Modulen wechselwirkt. Für Softwarekomponenten und Funktionen beinhaltet das logische und physikalische Zusammenhänge der intelligenten Informationsverarbeitung. Bei Hardwarekomponenten sind beispielsweise kinematische und geometrische Wirkungsketten sowie physische Aspekte von Bedeutung. Auf Basis dieser Komponentenstrukturen, die Top-Down aus dem Wechselspiel von Anforderungen und Strukturen abgeleitet wurden, erfolgen Detailentwurf und Test der Hard- und Softwarekomponenten im Rahmen der mechatronischen Komposition. Das Entwicklungsergebnis wird gegen die Anforderungen auf der Komponentenebene abgesichert. Ist der Mindesterfüllungsgrad der Anforderungen erreicht, kann das System sukzessive und Bottom-Up zum Gesamtsystem integriert und auf Systemebene validiert werden. Der Absicherungsprozess im Rahmen der MBRE-basierten mechatronischen Entwurfsmethodik ist iterativ. Bleiben gestellte oder abgeleitete Anforderungen unerfüllt, müssen die Mängel durch einen erneuten Durchlauf der Methodik ab dem betreffenden Punkt korrigiert werden. Erfüllt das SOI auf der Systemebene die definierten Anforderungen kann ein Akzeptanztest für die zu Beginn definierten Anwendungsfälle des SOI in seiner Umgebung stattfinden. Hieraus lassen sich Rückschlüsse auf den Erfüllungsgrad der Kunden- bzw. Stakeholderbedürfnisse und etwaige Konsequenzen ziehen.

Die Durchgängigkeit der MBRE-basierten mechatronischen Entwurfsmethodik wird durch die Pfeile in Ab- bildung 2 verdeutlicht. Ermöglicht wird sie durch die logische und modellbasierte Vorgehensweise mit der softwaretechnischen Umsetzung im Systemmodell. Die Entwurfsmethodik illustriert und gewährleistet die gegenseitige Abhängigkeit und Beeinflussung von Anforderungen, Struktur und Funktion des SOI. Aufgrund der Verknüpfung dieser Perspektiven und Systembestandteile, wird auch das automatische Übergeben und Anpassen dynamischer Anforderungen und Strukturen abgebildet. Voraussetzung hierfür ist eine entsprechende parametrische Implementierung in SysML. Darüber hinaus sind Ansätze zur Toolkopplung von SysML und den konkreten Entwicklungstools denkbar. So können im Systemmodell dokumentierte und verwaltete Anforderungen, Strukturen, Parameter oder Ergebnisse toolübergreifend genutzt werden.

\section{Validierung anhand der kon- fliktfreien Trajektorienplanung}

\subsection{Beschreibung des Anwendungsbeispiels}

Die hergeleitete ganzheitliche Entwurfsmethodik für die modellbasierte Entwicklung mechatronischer Systeme im digitalisierten und vernetzten Umfeld, soll anhand eines Anwendungsbeispiels exemplarisch demonstriert und validiert werden. Das Beispiel stammt aus dem Bereich der Fertigungsindustrie. Der Schlüssel zum Erhalt der Wettbewerbsfähigkeit sind neue I4.0-Technologien zur Flexibilitätssteigerung. [9] Fahrerlose Transportfahrzeuge (FTF) sind essentielle Bestandteile einer intelligenten Fabrik und müssen ebenfalls zur Erfüllung der Flexibilitätsanforderungen beitragen. Daher wird an der Ostfalia Hochschule ein intelligentes, autonom agierendes und bewegungsflexibles FTF entwickelt und die zugehörigen Funktionen modellbasiert ausgelegt.

So auch eine Funktion zur konfliktfreien, selbstoptimierten Trajektorienplanung, welche in [10] detailliert beschrieben wird. In diesem Beitrag soll der Entwurfsprozess anhand der vorgestellten Entwurfsmethodik vom Anforderungsmanagement bis hin zur Validierung beschrieben werden.

\subsection{Anwendung der MBRE-basierten mechat- ronischen Entwurfsmethodik}

Ausgangspunkt des Anwendungsbeispiels ist die Stakeholderebene, auf der übergeordnete, abstrakte Wünsche und Bedürfnisse der Fabrikleitung, wie der Erhalt der 
Wettbewerbsfähigkeit, Flexibilität oder die Verbesserung des logistischen Flusses liegen. Denkbare Anwendungsfälle die sich hierfür definieren lassen, zielen auf ein flexibles Produktionssystem mit hohem Automatisierungsgrad ab. Neben reinen Fertigungstätigkeiten ist hierfür auch das Intralogistikmanagement entscheidend. Aus den Anwendungsfällen werden die Interaktionen des FTF als SOI, mit seiner Umgebung, z.B. Maschinen, Lagersystemen oder dem internen Verkehrssystem deutlich. Hieraus lassen sich Anforderungen ableiten und neu definieren. Beispielsweise muss sich das FTF an die zulässige Höchstgeschwindigkeit von $1 \mathrm{~m} / \mathrm{s}$ halten. Konstruktionstechnische Anforderungen ergeben sich zusätzlich z.B. aus dem Grundriss sowie der verfügbaren Spurbreite von $0,5 \mathrm{~m}$ auf den Fabrikstraßen oder auch der Geometrie der Transportgüter. Des Weiteren müssen drahtlose Kommunikationstechnologien und -standards vorgesehen werden, damit das FTF mit seiner Umgebung, beispielsweise anderen FTF, dem Intralogistik-, Warenhausoder Instandhaltungsmanagement kommunizieren kann. Aus der Rolle des FTF in seinem Systemkontext ergeben sich im nächsten Schritt Aufgaben bzw. Anforderungen auf der Systemebene. Einige dieser Anforderungen, wie das Einhalten zulässiger Geschwindigkeiten oder die Abmaße des FTF $(B \times L=0,2 \mathrm{~m} \times 0,3 \mathrm{~m})$ werden direkt Top-Down abgeleitet. Andere ergeben sich indirekt durch Vorgaben oder Restriktionen auf Systemkontextebene. Um beispielsweise den Anforderungen an Flexibilität in der engen Produktionsumgebung gerecht zu werden, muss sich das FTF losgelöst von vorgegebenen Spuren und omnidirektional bewegen können. Daraus wiederum resultiert, dass das FTF zur selbstständigen Navigation z.B. eine Trajektorienplanung benötigt. Des Weiteren ist es möglich auf der Systemebene neue Anforderungen zu definieren. Beispielsweise soll das FTF Kollisionen mit anderen FTF nicht erst lokal, sondern bereits während der Routenplanung mit einer Konflikterkennungs- und Lösungsfunktion vermeiden. Führt man dieses Vorgehen fort und ordnet die sich ergebenden Anforderungen und Systembestandteile hierarchisch an, entsteht die mechatronische Strukturierung. Diese wurde gemeinsam mit den parametrischen dynamischen Anforderungen in SysML implementiert und ist exemplarisch in Abbildung 3 dargestellt.

Die SysML ermöglicht die Darstellung von Strukturund Verhaltensdiagrammen, wie den Blockdefinitionsdiagrammen aus Abbildung 3. Sie bietet darüber hinaus den Diagrammtyp der Anforderungsdiagramme. Das Anforderungsdiagramm wird zunächst als separates Diagramm erstellt und anschließend den Strukturebenen und -elementen zugeordnet. Bei der Beziehung der Anforderungen untereinander besteht ein Unterschied, ob sich beispielsweise eine Systemanforderung von einer Systemkontextanforderung ableiten lässt (derive) oder eine Anforderung sich aus mehreren Anforderungen zusammensetzt (containment). Um die Beziehung zwischen einem Strukturelement und einer Anforderung zu beschrei-

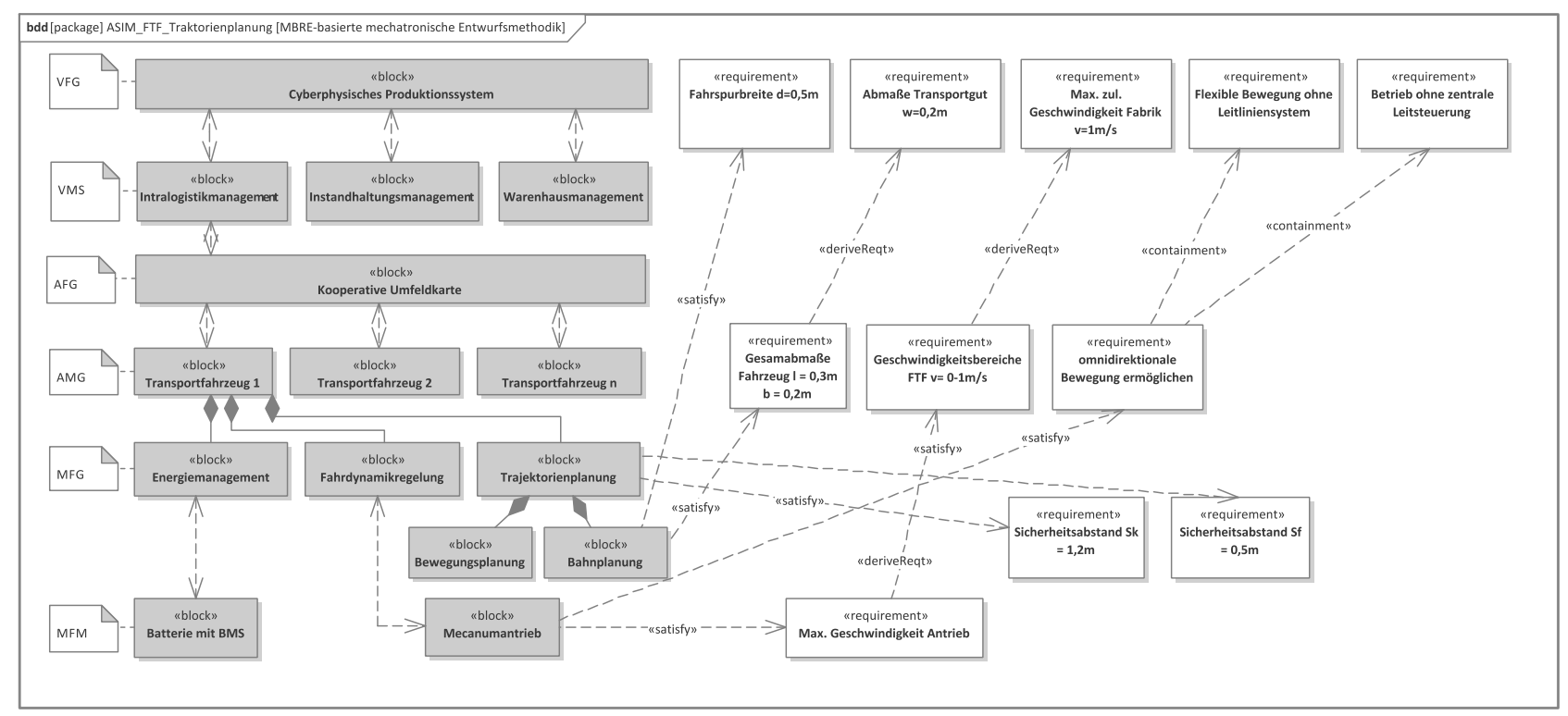

Abbildung 3: Exemplarisches Blockdefinitionsdiagramm der mechatronischen Strukturierung des FTF im CPS mit den verknüpften Anforderungen in SysML 
ben, existiert die "satisfy"-Beziehung. Zur Verdeutlichung dieser Zusammenhänge, wurde eine Auswahl der Anforderungen direkt in das Blockdefinitionsdiagramm in Abbildung 3 implementiert.

Anschließend werden Anforderungen auf Komponentenebene abgeleitet und definiert. Da sich dieses Anwendungsbeispiel mit der konfliktfreien Trajektorienplanung befasst, wird diese exemplarisch vorgestellt. Um eine Kollision in einem Kreuzungskonflikt zu vermeiden, muss beispielsweise ein Sicherheitsabstand $S$ zwischen den FTF eingehalten werden. Dieser berechnet sich zu $S=f\left(v_{\text {max }}, G, d\right)$ in Abhängigkeit der maximalen Geschwindigkeit $v_{\max }$ der FTF, dessen Geometrie $G$ und der verfügbaren Fahrspurbreite $d$.

Mit den restlichen Informationen aus dem Systemmodell kann man für das Modul Trajektorienplanung die in Abbildung 4 dargestellte Funktionsstruktur aufstellen, die alle Wirkungszusammenhänge, Schnittstellen und das Verhalten dieser Funktion vom Erhalt eines Auftrags, über die Zielführung, Trajektorienberechnung und Konfliktlösung bis hin zur Fahrdynamikregelung beschreibt. Nach Abbildung 2 folgt nun der Detailentwurf und Test der Komponente bzw. die sukzessive Integration.

Die modellbasierte Auslegung des Funktionsmoduls zur Trajektorienplanung sowie die Simulationsergebnisse bei dynamischen Anforderungen werden im folgenden Abschnitt vorgestellt.

\subsection{Auswertung der Simulationsergebnisse}

Die Funktion zur Trajektorienplanung wurde auf Basis der hergeleiteten Funktionsstruktur nach Abbildung 4 unter Anwendung der in diesem Beitrag erarbeiteten MBRE-basierten mechatronische Entwurfsmethodik ausgelegt. Die Auswertung der Simulationsergebnisse soll den durchgängigen Charakter der Entwurfsmethodik und deren Vorteil im Umgang mit dynamischen Anforderungen exemplarisch zeigen. Aus den Anforderungen für Kreuzungskonflikte ergibt sich für die ursprüngliche Konfiguration von $v_{\max }, G$ und $d$ ein Mindestsicherheitsabstand von $S=1,2 \mathrm{~m}$. Ein solcher Konflikt wird gelöst,

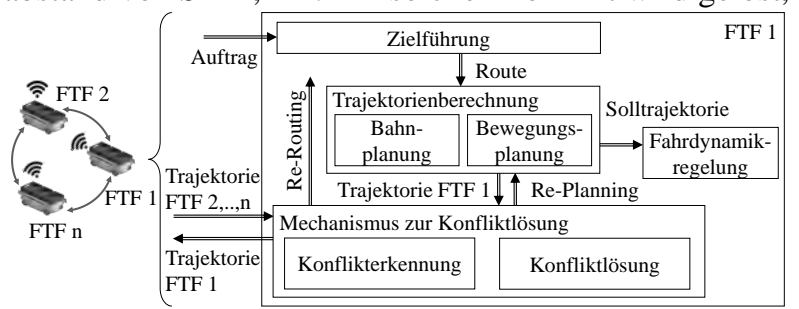

Abbildung 4: Funktionsstruktur der konfliktfreien Trajektorienplanung indem das FTF mit dem niedriger priorisierten Auftrag seine Geschwindigkeit reduziert. Abbildung 5 zeigt ein Simulationsergebnis, bei dem ein Kreuzungskonflikt vorliegt. Das FTF1 hat in diesem Fall seine Geschwindigkeit so reduziert, dass der kleinste Abstand zwischen den FTF 1,4 m beträgt. Das bedeutet, dass die Funktion so ausgelegt wurde, dass die Anforderung an den Mindestabstand erfüllt wurde.

Nun wird angenommen, dass die Fabrikleitung die zulässige Geschwindigkeit aus Sicherheitsgründen auf $0,6 \mathrm{~m} / \mathrm{s}$ reduziert. Durch die parametrische Implementierung der dynamischen Anforderungen im Systemmodell, wird diese Änderung automatisch an die unterlagerten und verknüpften Anforderungen und Systembestandteile weitergeleitet. Aufgrund dessen beträgt $S$ nun nur noch 0,6 m. Die Kopplung dieser Anforderung mit den Parametern des modellbasierten Detailentwurfs erlaubt eine einfache Aktualisierung der Funktion. Ein entsprechendes Simulationsergebnis ist in Abbildung 6 illustriert. Es ist zu sehen, dass das FTF1 seine Geschwindigkeit um ein geringeres Maß reduziert. Der Mindestabstand liegt mit 0,7 m immer noch über dem geforderten Wert.

Dieses Anwendungsbeispiel demonstriert den ganzheitlichen und durchgängigen Charakter der MBRE-basierten Entwurfsmethodik. Es zeigt, dass durch die Kopplung von MBRE und mechatronischer Entwurfsmethodik ein echter Mehrwert entsteht, der aus den Abhängigkeiten und Wechselwirkungen von Anforderungen, Struktur und Funktionen eines Systems resultiert. Die Sinnhaftigkeit und Funktionsfähigkeit dieses Ansatzes wurde in dieser Anwendung von abstrakten übergeordneten Anforderungen bis zum Detailentwurf und Test validiert.

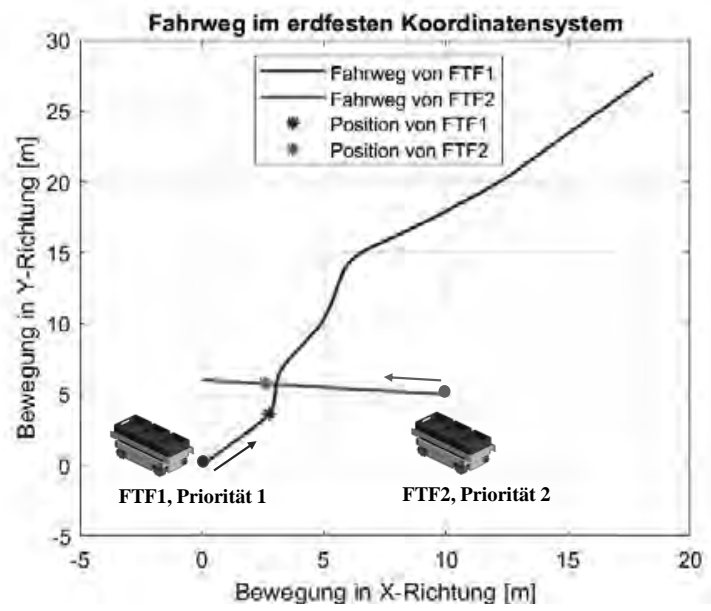

Abbildung 5: Simulation eines Kreuzungskonflikts mit $\mathrm{v}_{\max }=1 \mathrm{~m} / \mathrm{s}$ und $\mathrm{S}=1,2 \mathrm{~m}$ 


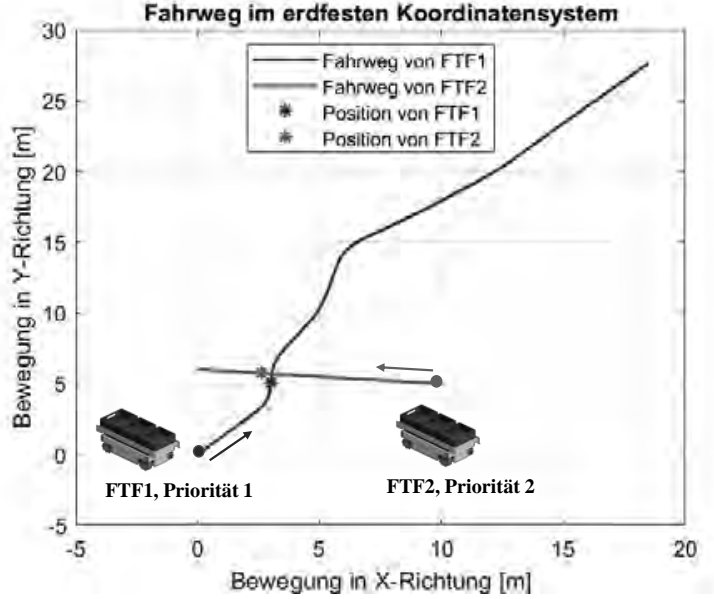

Abbildung 6: Simulation eines Kreuzungskonflikts mit $\mathrm{V}_{\max }=0,6 \mathrm{~m} / \mathrm{s}$ und $\mathrm{S}=0,6 \mathrm{~m}$

\section{Zusammenfassung und Ausblick}

Anforderungsmanagement und mechatronischer Entwurf bieten unterschiedliche Perspektiven und Schwerpunkte, sind aber beide essentiell für die Entwicklung komplexer Systeme. Das MBRE beschreibt Systemzusammenhänge und Anforderungen auf einer übergeordneten Metaebene, wohingegen der mechatronische Entwurf funktionale und strukturelle Wechselwirkungen des Systems fokussiert. Die MBRE-basierte mechatronische Entwurfsmethodik ermöglicht eine ganzheitliche Betrachtung von Anforderungen, Struktur, Funktionen und Absicherung des SOI. Aufgrund der Verknüpfung dieser Perspektiven und Systembestandteile, wird auch das automatische Übergeben und Anpassen dynamischer Anforderungen und Strukturen unterstützt.

Anhand der konfliktfreien Trajektorienplanung konnte das Vorgehen aufgezeigt und die Durchgängigkeit und Funktionsfähigkeit der Methodik mit Simulationen demonstriert und abgesichert werden.

Ausblickend sind weitere Arbeiten zur Kopplung von SysML-basierten und beispielsweise nummerischen Entwicklungstools denkbar. Somit ließe sich eine interdisziplinäre Entwicklungsumgebung realisieren, in der ausgehend von den im Systemmodell dokumentierten und verwalteten Anforderungen, auch Strukturen, Parameter oder Ergebnisse in tool-übergreifenden Anwendungen genutzt werden können.

\section{Danksagung}

Diese Publikation wurde gefördert durch:

- Europäischer Fond für regionale Entwicklung (EFRE): Verbundprojekt "autoMoVe" (Dynamisch konfigurierbare Fahrzeugkonzepte für den nutzungsspezifischen autonomen Fahrbetrieb) (ZW 685030889)

- EFRE: Verbundprojekt "Synus" (Methoden und Werkzeuge für die synergetische Konzipierung und Bewertung von Industrie 4.0-Lösungen) (ZW 6-85012454)
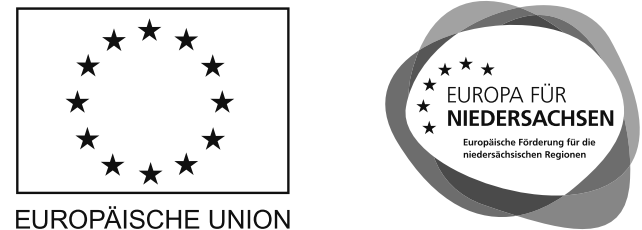

\section{Literatur}

[1] Czichos, H. Mechatronik. Springer Vieweg, Wiesbaden, 2019.

[2] Huth T, Vietor T. Systems Engineering in der Produktentwicklung: Verständnis, Theorie und Praxis aus ingenieurswissenschaftlicher Sicht. Gruppe. Interaktion. Organisation. Zeitschrift für Angewandte Organisationspsychologie (GIO), Jg. 51, Nr. 1, S. 125-130, 2020.

[3] Haberfellner, R., Daenzer, W.F.: Systems Engineering: Methodik und Praxis. Verl. Industrielle Organisation, Zürich (1999)

[4] Friedenthal, S. and Oster, C. Architecting spacecraft with SysML: A model-based systems engineering approach, CreateSpace Independent Pub. Platform, s.l..,2017.

[5] Pohl K., Rupp C.Basiswissen Requirements Engineering, dpunkt.verlag 2015.

[6] Gilz T. Requirements Engineering und Requirements Management in Modellbasierte virtuelle Produktentwicklung, S 59. Springer, Heidelberg 2014.

[7] Liu-Henke X. Mechatronische Entwicklung der aktiven Feder-Neigetechnik für das Schienenfahrzeug RailCab. VDI Fortschritt-Berichte, Reihe 12, Verkehrstechnik / Fahrzeugtechnik, Nr. 589. Düsseldorf: VDI-Verlag 2005.

[8] Liu-Henke X, Yarom OA, Scherler S. Virtual Development and Validation of a Function for an Automated Lateral Control using Artificial Neural Networks and Genetic Algorithms. 91st IEEE Vehicular Technology Conference (VTC2020-Spring), Antwerpen, Belgien, 25. 28. Mai, 2020.

[9] Bauernhansel T, Hompel M, Volgel-Heuser B, Industrie 4.0 in Produktion, Automatisierung und Logistik, Springer Vieweg, Münschen, 2014.

[10] Zhang J., Liu-Henke X. Konfliktfreie, selbstoptimierte Trajektorienplaung für ein fahrerloses Transportfahrzeug zur Durchführung des autonomen Gütertransportes im Produktionsumfeld, 25. Symposium Simulationstechnik - ASIM 2020 - Virtuelle Tagung, 14 -15. Oktober, 2020. (tbp) 